\title{
Agenesis of the dorsal pancreas: a rare cause of diabetes
}

\author{
Masanori Shimodaira • Naoko Kumagai • \\ Erisa Sorimachi $\cdot$ Mitsuhiko Hara • \\ Kazufumi Honda
}

Received: 2 April 2011/Accepted: 28 April 2011/Published online: 15 May 2011

(c) SIMI 2011

\section{Case report}

A 19-year-old Japanese man visited our department presenting with glycosuria that had been detected during a routine school urinalysis. The patient had undergone cardiac surgery at the age of 4 years for atrial and ventricular septal defects, and pulmonary artery stenosis. He denied any history of abdominal trauma, drug or alcohol intake, or gastrointestinal problems. His maternal grandmother had mild diabetes that began in middle age; whereas, his parents had no significant medical history including diabetes.

The physical examination revealed an emaciated young man with a body mass index of 19.3 , but otherwise a normal examination. Laboratory studies were: fasting hyperglycaemia of $19.7 \mathrm{mmol} / \mathrm{l}(354 \mathrm{mg} / \mathrm{dl})$ without ketosis. The HbAlc was $12.1 \%$, urinary excretion of C-peptide $43.9 \mu \mathrm{g} /$ day (normal range 50-100 $\mu \mathrm{g} / \mathrm{day}$ ). The anti-islet cell and anti-glutamic acid decarboxylase antibodies were negative. The serum total amylase level was normal, $49 \mathrm{U} / 1$ (normal range 40-180 U/l); whereas, the levels of serum pancreatic enzymes p-type amylase, lipase, trypsin and phospholipase $\mathrm{A}_{2}$ were decreased to $8 \mathrm{U} / 1$ (normal range $17-50 \mathrm{U} / \mathrm{l}), 10 \mathrm{U} / 1$ (13-49 U/l), $50 \mathrm{U} / 1(100-550 \mathrm{U} / \mathrm{l})$ and $100 \mathrm{U} / 1$ (130-400 U/l), respectively.

Ultrasonography (US) of the abdomen could not identify the body of the pancreas, although the head and uncus were

M. Shimodaira $(\varangle) \cdot$ N. Kumagai · E. Sorimachi $\cdot$ K. Honda Department of Endocrinology and Metabolism, Tokyo Metropolitan Hiroo Hospital, 2-34-10 Ebisu, Shibuya-ku, Tokyo 150-0013, Japan

e-mail: masanori19810813@yahoo.co.jp

M. Hara

Department of Pediatrics, Tokyo Metropolitan Hiroo Hospital, Tokyo, Japan clearly visible. Computed tomography (CT) and magnetic resonance imaging (MRI) confirmed that most of the body and the tail of the pancreas were absent, and that the pancreatic head was slightly enlarged (Fig. 1). Magnetic resonance cholangiopancreatography (MRCP) revealed that the dorsal pancreatic duct was absent. Based on these findings, a diagnosis of agenesis of the dorsal pancreas was made. Insulin replacement therapy was initiated, and currently, the diabetes is well controlled with $4 \mathrm{U}$ of insulin per day. There are no signs of any diabetes-related renal, retinal or neurological changes. His blood glucose levels have improved after beginning insulin therapy, and he is being followed up as a hospital outpatient.

\section{Discussion}

Developmental anomalies of the pancreas are rarely seen. Pancreas divisum is the most common anomaly. However, agenesis of the dorsal pancreas, with the absence of the pancreatic body and tail is very rare; only 53 cases have been described in medical literature in the last 100 years [1].

The embryology of the pancreas is complex. During gastrulation, the epiblast comprised multipotential cells that differentiate into three embryonic layers: ectoderm, endoderm and mesoderm. The gastrointestinal organs, including the pancreas, are derived from the endoderm. The pancreas develops embryologically from a dorsal and a ventral bud on opposite sides of the foregut. Morphogenetically, the ventral pancreatic bud goes on to form the posterior part of the head, and the dorsal pancreatic bud contributes mainly to the formation of the tail and body. During the growth and the rotation of the gut tube in the seventh week of gestation, the two buds fuse. As these two buds grow, each bud develops a tree-like ductal system. 

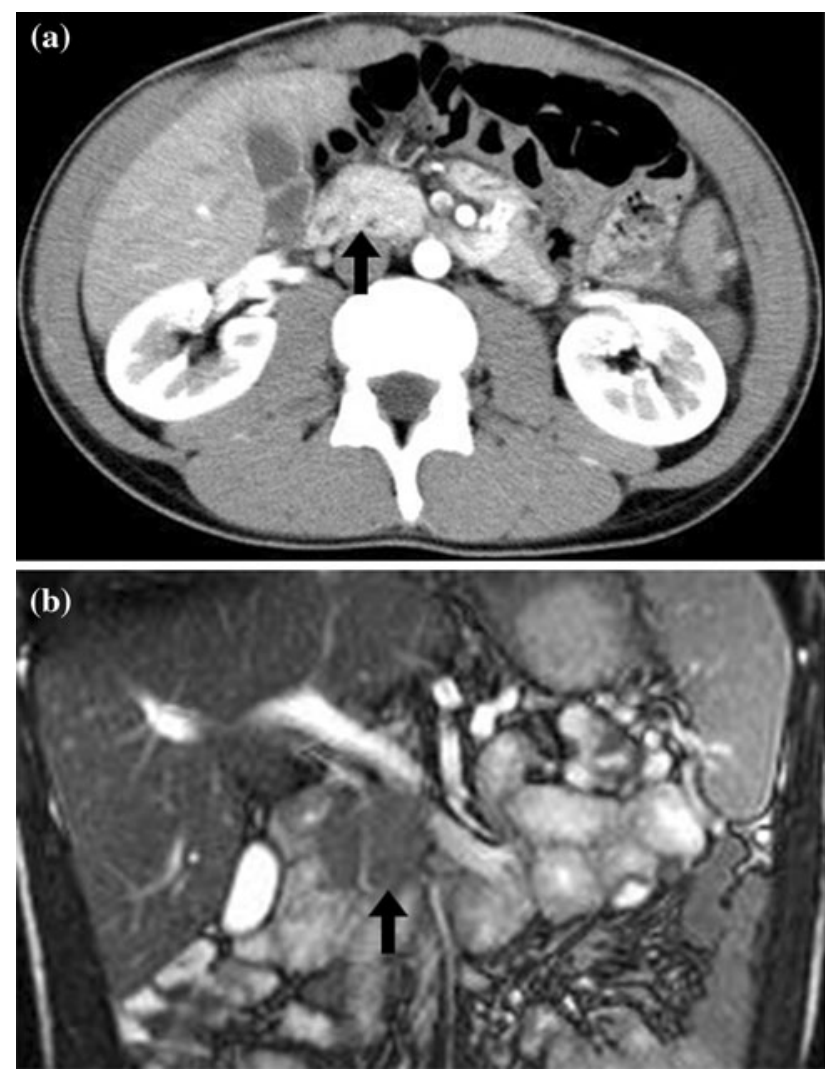

Fig. 1 Axial CT image (a) and coronal MRI image (b) showing a slightly hypertrophic pancreatic head, but with non-visualization of the body and tail of the pancreas

Agenesis of the dorsal pancreas is usually detected by imaging studies performed during clinical assessment of a patient with abdominal pain. Non-specific abdominal pain is the most common presenting symptom of dorsal pancreatic agenesis. However, its association with pancreatitis is controversial and the mechanism underlying the associated pancreatitis is speculative. Several mechanisms such as sphincter of Oddi dysfunction and dyskinesia accompanied by compensatory hypertrophy and hypersecretion of the remaining ventral pancreas with raised intraductal pressure have been proposed to explain the pancreatitis [2]. In addition to abdominal pain, it is reported that approximately $50 \%$ of patients with agenesis of the dorsal pancreas are hyperglycaemic [1] because the majority of islets are located in the pancreatic tail, and $\beta$-cells of the dorsal pancreas respond positively to glucose stimulation. In addition to a decrease in $\beta$-cell mass, insulin resistance is also the root cause in the most common forms of diabetes. In our case, a year before, the patient was diagnosed with diabetes, he ate excessively due to the stress of a university entrance examination. His irregular dietary habits might have contributed to the development of insulin resistance, which ultimately accelerated the development of diabetes.
Agenesis of the dorsal pancreas has also been reported as a part of polysplenia syndrome. The concomitant anomalies in this syndrome may be related to the proximity of the developing pancreas and spleen in the dorsal mesogastrium [3]. Moreover, the association of congenital heart defects such as septal defects, tetralogy of Fallot or pulmonary artery stenosis is common [4]. Although few cases of agenesis of the dorsal pancreas describe features similar to our patient, the individual symptoms may vary from case to case [1]. Most cases describe single, but not familiar, patients. In our patient, neither his maternal grandmother, who had mild diabetes, nor his mother, who was non-diabetic had the anomaly.

An accurate diagnosis of agenesis of the dorsal pancreas is confronted by technical problems. Abdominal ultrasound imaging (US) has limitations because of non-visualization of the body and tail of the pancreas due to interference from bowel gas or even technical failure. An MRI or CT scan can facilitate diagnosis by demonstrating the absence of the pancreatic body and tail, and the presence of the pancreatic head. Only endoscopic retrograde pancreatography and MRCP help in the demonstration of the missing dorsal pancreatic duct and permit correct diagnosis of agenesis of the dorsal pancreas [5]. With improvement in imaging studies, the diagnosis is being made with increased frequency.

\section{Conclusion}

Agenesis of dorsal pancreas is a rare condition, but may be worth searching for in young patients with diabetes mellitus having a history of other congenital anomalies.

\section{Conflict of interest None.}

\section{References}

1. Schnedl WJ, Piswanger-Soelkner C, Wallner SJ, Reittner P, Krause R, Lipp RW, Hohmeier HE (2009) Agenesis of the dorsal pancreas and associated diseases. Dig Dis Sci 54:481-487

2. Rakesh K, Choung OW, Reddy DN (2006) Agenesis of the dorsal pancreas (ADP) and pancreatitis is there an association? Indian $\mathrm{J}$ Gastroenterol 25:35-36

3. Herman TE, Siegel MJ (1991) Polysplenia syndrome with congenital short pancreas. Am J Roentgenol 156:799-800

4. Balasubramanian M, Shield JP, Acerini CL, Walker J, Ellard S, Marchand M, Polak M, Vaxillaire M, Crolla JA, Bunyan DJ, Mackay DJ, Temple IK (2010) Pancreatic hypoplasia presenting with neonatal diabetes mellitus in association with congenital heart defect and developmental delay. Am J Med Genet A 152A: 340-346

5. Macari M, Giovanniello G, Blair L, Krinsky G (1998) Diagnosis of agenesis of the dorsal pancreas with MR pancreatography. Am J Roentgenol 170:144-146 